\title{
Primary malignant CNS lymphoma in an immunocompetent patient: A case report
}

\author{
Khaled A', Salauddin $\mathrm{SA}^{2}$, Zaman $\mathrm{SMQ}^{3}$, Nasir $\mathrm{TA}^{4}$
}

\begin{abstract}
Aim and Objective: Primary malignant CNS lymphoma in otherwise healthy immunocompetent subject is relatively uncommon. They occur more frequently in immunocompromised patients. In this case report, we presented a report of an immunocompetent 48 year old male with primary CNS lymphoma

Clinical presentation: A 48 year old male was admitted in the neurosurgery department of Apollo hospital Dhaka because of continuous headache and vomiting for 15 days. There was no history of any illness, medication or head trauma. He was tested negative for HIV test. MRI revealed a periventricular mass which was confirmed as primary CNS lymphoma, diffuse large cell type by histopathology and immunohistochemical examination.
\end{abstract}

Conclusion: We presented this case because of relatively uncommon occurrence and raising incidence of primary CNS lymphoma in immunocompetent subjects in last one or two decades.

Key Word: Brain tumor, CNS lymphoma, immunocompetent

Introduction: Primary CNS lymphoma is extranodal malignant lymphomas arising in the CNS in the absence of obvious lymphoma outside the nervous system at the time of diagnosis. ${ }^{1}$ Primary CNS lymphomas arise sporadically in otherwise healthy subjects but occur more frequently in the immunologically compromised particularly those with HIV infection and post transplant patient. ${ }^{2}$ Primary CNS lymphoma affects all ages with a peak incidence in immunocompetent subjects during the 6th and 7 th decades and usually 3rd and 4th decade in immunocompromised patient. About $60 \%$ of primary CNS lymphoma involve the supratentorial space including the frontal $(15 \%)$, temporal $(8 \%)$, parietal $(17 \%)$, occipital $(3 \%)$, basal ganglia/ periventricular region $(10 \%)$, corpus callosum (5\%), posterior fossa $(13 \%)$ and the spinal cord $(1 \%){ }^{1}$

Case report: A 48 year old male was admitted in the neurosurgery department of Apollo hospital Dhaka because of continuous headache with associated vomiting for 15 days. There was no history of febrile illness, head trauma, recent diagnosis of cancer, medication or any other illness. He also did not give any history of loss of consciousness or seizures. His physical examination revealed signs of raised intracranial pressure and both pupil were non reactive to light with decreased Glassgow coma scale (E4V4M6). Muscle power and other vitals were within normal limit. All laboratory examination was within normal range and he was negative for HIV test. MRI with contrast showed multiple well enhancing periventricular mass lesions with edema and mass effect suggestive of lymphoma. Left occipital craniotomy followed by debulking of tumor was done and tissue was sent for histopathological examination. Grossly, there were multiple grayish white pieces of tissue measuring between $3 \times 2.5 \times 2 \mathrm{~cm}$ and $1 \times 0.8 \times 0.5 \mathrm{~cm}$. Microscopic examination showed diffuse infiltration of atypical large lymphoid appearing cells in the brain parenchyma with marked perivascular cuffing (angiocentric pattern) (Fig:1).

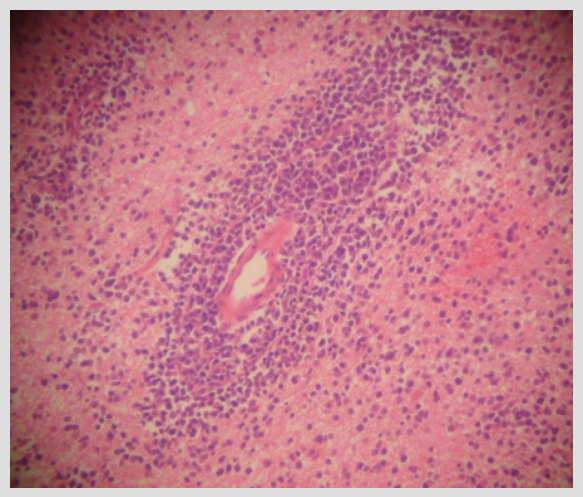

Figure 1: Diffuse infiltration of atypical large lymphoid cells. In the brain parenchyma with angiocentric pattern

Immunohistochemically, the neoplastic cells were strongly positive for leukocyte common antigen (LCA) (Fig:2) and a diagnosis of primary malignant CNS lymphoma, diffuse large cell type were made.

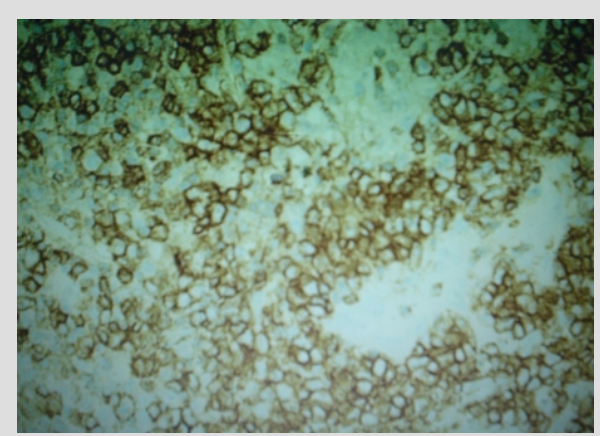

Figure 2: Tumor cells showing strong immuoreactivity for Leucocyte Common Antigen(LCA) 


\section{Primary malignant CNS lymphoma}

Discussion: It is not known whether primary CNS lymphoma arise within or outside the brain and why they manifest in an organ that lacks a regular lymphatic system. Three hypothesis have been put forward (1) B cells may be transformed at a site elsewhere and then develop adhesion molecules specific for cerebral endothelia (2) lymphoma cells may be systemically eradicated by an intact immune system but may be relatively protected within the CNS (3) A polyclonal intracerebral inflammatory lesion may expand clonally and may progress to the monoclonal neoplastic state. ${ }^{1}$

Epstein-Barr virus play's a major role in the etiology of CNS lymphoma in case of immunocompromised patients but it has minor role in immunocompetent cases. ${ }^{1}$

In an extensive study by Paul and his team on 56 Indian populations for a period of 20 years, they found median age of onset was 41 years, one patient was HIV positive with rest of them immunocompetent and histopathologically all the cases were diffuse large cell lymphoma. ${ }^{3}$ Their findings are almost similar to this presenting case. Over the last decade, the incidence of primary CNS lymphoma reported in most studies has risen threefold, which cannot be entirely explained by the increased prevalence of AIDS, or the growing number of organ transplantation. Incidence grew significantly not only in immunosuppressed patients, but also in immunocompetent patients. ${ }^{4} \mathrm{Mi}$ and $\mathrm{Mittal}^{5}$ reported a case of 77 year old immunocompetent women with cognitive decline in whom a tentative diagnosis of primary CNS lymphoma was made. Brunnstrom et $\mathrm{al}^{6}$ also reported an immmunocompetent 76 year old patient with cognitive and neurological symptoms.

The treatment of primary CNS lymphoma includes chemotherapy and radiotherapy. ${ }^{7}$ With this therapy immunocompetent patients show response rate of $85 \%$ with a median survival of 17-45 months and immunocompromised patients 2-6 months. Some authors have also found that histological subtypes have a direct correlation with survival. ${ }^{8}$

In conclusion, we presented herein a report of an immunocompetent 48 year old male with primary CNS lymphoma. Pathological examination and immunohistochemistry provide the diagnosis of diffuse large cell lymphoma.

References:

1. Paulus W, Jellinger K, Morgello S, Schluter MD. Malignant lymphomas. In Kleihues P, Cavenee WK, editors. Pathology and Genetics of Tumours of the Nervous System. Lyon: IARC Press; 2000. p. 198-203.

2. Burger PC, Scheithauer BW. Tumors of the Central Nervous System. Atlas of Tumor Pathology, 3rd series. Washington, DC: Armed Forces Institute of Pathology;1994. p. 321-327.

3. Paul TR, Challa S, Tandon A, Panigrahi MK1, Purohit AK. Primary central nervous system lymphomas: indian experience, and review of literature. Indian Journal of Cancer. 2008;45(3):112-118.

4. Hong KS, Kim SD, Lim DJ, Park JY. Primary central nervous system lymphoma in organ recipient. J of Korean Neurosurgery. 2006;37:296299. J

5. Mi W, Mittal S. Primary lymphoma of the central nervous system in an immunocompetent patient. Hospital Physician. 2001;34-38.

6. Brunnstrom H, Dictor M, Nilsson C, Gulich D, Englund E. A 76 year old man with cognitive and neurologic symptoms. Brain Pathol. 2009;19(4):731-4.

7. Obrein PC, Roose DE, Liew KH, Trotter GE, Barton MB, Walker QJ. Preliminary results of combined chemotherapy and radiotherapy for non-AIDS primary central system lymphoma. MED J Aus. 1996; 165:424-427.

8. Miller DC, Hochberg FH, Harris NL, Gruber ML, Louis DN. Pathology with clinical correlation of primary central nervous system non-hodgkins lymphoma. The Massachusetts General Hospital experience 1958-1989. Cancer. 1994;74:1383-1397. 